\title{
Localization of Energy-Momentum for a Black Hole Spacetime Geometry with Constant Topological Euler Density
}

\author{
Irina Radinschi $\left(\mathbb{D},{ }^{1}\right.$ Theophanes Grammenos, ${ }^{2}$ Farook Rahaman $\mathbb{D}^{\mathrm{D}},{ }^{3}$ Andromahi Spanou, ${ }^{4}$ \\ Marius Mihai Cazacu, ${ }^{1}$ Surajit Chattopadhyay, ${ }^{5}$ and Antonio Pasqua $\mathbb{D}^{6}$ \\ ${ }^{1}$ Department of Physics "Gh. Asachi” Technical University, Iasi 700050, Romania \\ ${ }^{2}$ Department of Civil Engineering, University of Thessaly, 38334 Volos, Greece \\ ${ }^{3}$ Department of Mathematics, Jadavpur University, Kolkata 700 032, West Bengal, India \\ ${ }^{4}$ School of Applied Mathematics and Physical Sciences, National Technical University of Athens, 15780 Athens, Greece \\ ${ }^{5}$ Department of Mathematics, Amity University, Kolkata 700135, India \\ ${ }^{6}$ Department of Physics, University of Trieste, 34127 Trieste, Italy
}

Correspondence should be addressed to Irina Radinschi; radinschi@yahoo.com

Received 5 July 2018; Revised 1 October 2018; Accepted 16 October 2018; Published 25 October 2018

Academic Editor: Elias C. Vagenas

Copyright (C) 2018 Irina Radinschi et al. This is an open access article distributed under the Creative Commons Attribution License, which permits unrestricted use, distribution, and reproduction in any medium, provided the original work is properly cited. The publication of this article was funded by $\mathrm{SCOAP}^{3}$.

\begin{abstract}
The evaluation of the energy-momentum distribution for a new four-dimensional, spherically symmetric, static and charged black hole spacetime geometry with constant nonzero topological Euler density is performed by using the energy-momentum complexes of Einstein and Møller. This black hole solution was recently developed in the context of the coupled Einstein-nonlinear electrodynamics of the Born-Infeld type. The energy is found to depend on the mass $M$ and the charge $q$ of the black hole, the cosmological constant $\Lambda$, and the radial coordinate $r$, while in both prescriptions all the momenta vanish. Some limiting and particular cases are analyzed and discussed, illustrating the rather extraordinary character of the spacetime geometry considered.
\end{abstract}

\section{Introduction}

The issue of energy-momentum localization systematised researchers' work in a special way. Looking deeply into the problem, it was clear that the main difficulty consists in the lack of a proper definition for the energy density of gravitational backgrounds. In this light, much research work has been done over the last years concerning the best tools used for the energy-momentum localization. A brief survey points out the leading role played notably by super-energy tensors [1-4], quasi-local expressions [5-10], and the famous energy-momentum complexes of Einstein [11, 12], Landau-Lifshitz [13], Papapetrou [14], BergmannThomson [15], Møller [16], and Weinberg [17]. Among the aforementioned computational tools, the energy-momentum complexes have been proven to be interesting and useful as well due to the diverse and numerous reasonable expressions that can be obtained by their application. Some observations are in order here. First, according to their underlying mathematical mechanism, their construction involves the use of two parts, one for the matter and the other for the gravitational field. Second, despite the fact that the energymomentum complexes allow one to obtain many interesting and physically meaningful results for different space-time geometries, their construction is connected to an inherent central problem, namely, their coordinate dependence. As it is well-known from the relevant literature, this problem has found a solution in the case of the Møller energymomentum complex. Indeed, the calculations for the energymomentum of a given gravitational background in the Møller prescription enable the use of any coordinates such that the energy density component transforms as a four-vector density under purely spatial coordinate transformations for metrics with a line element of the form $d s^{2}=g_{00} d t^{2}-$ $g_{i j} d x^{i} d x^{j}$. As for the other energy-momentum complexes, the Schwarzschild Cartesian coordinates and the Kerr-Schild 
Cartesian coordinates have to be utilised for the calculations providing physically reasonable results for the cases of spacetime geometries in $(3+1),(2+1)$, and $(1+1)$ dimensions (see, e.g., [18-34] and references therein). At this point it should be noticed that, in order to avoid the coordinate dependence, an alternative method for the computation of the energy and momentum distributions is provided by the teleparallel equivalent of general relativity and certain modified versions of the teleparallel theory (see, e.g., [35-40] and references therein).

Regarding the Einstein, Landau-Lifshitz, Papapetrou, Bergmann-Thomson, Weinberg, and Møller energy-momentum complexes there is an agreement with the definition of the quasi-local mass introduced by Penrose [41] and developed by Tod [42] for some gravitational backgrounds. We point out that some rather recent works show that several energy-momentum complexes "provide the same results" for any metric of the Kerr-Schild class and indeed even for solutions that are more general than those of the KerrSchild class (see, e.g., [43, 44] and the interesting article [45] on the subject). Further, the entire historical development of the energy-momentum complexes that started with the formulation of their definitions also includes the attempts made for their rehabilitation [46-49]. In this sense, perhaps the most interesting issue was the fact that different energymomentum complexes yield the same results for the energymomentum distribution in the case of various gravitating systems.

The present paper has the following structure: in Section 2 we describe the new class of four-dimensional spherically symmetric, static, and charged black hole solutions with constant nonzero topological Euler density which we will consider. Section 3 focuses on the presentation of the Einstein and Møller prescriptions used for performing the calculations. In Section 4 we present the calculations and the results obtained for the energy and momentum distributions. Finally, in the discussion provided in Section 5, we give a brief description of the results obtained as well as some limiting and particular cases. Throughout we use geometrized units ( $c=G=1)$ and the signature chosen is $(+,-,-,-)$. Further, the calculations are performed by using the Schwarzschild Cartesian coordinates $\{t, x, y, z\}$ for the Einstein energymomentum complex and the Schwarzschild coordinates $\{t, r, \theta, \varphi\}$ for the Møller energy-momentum complex. Finally, Greek indices run from 0 to 3, while Latin indices range from 1 to 3 .

\section{Description of the New Black Hole Solution with Constant Topological Euler Density}

This section deals with the presentation of the new fourdimensional spherically symmetric, static, and charged black hole solution with constant topological Euler density [50] examined in the present study. Connecting geometry with topology, from the generalised Gauss-Bonnet theorem (see, e.g., [51]) applied to four dimensions the Euler-Poincaré characteristic is obtained by the integral of the Euler density

$$
\mathscr{G}=\frac{1}{32 \pi^{2}}\left(R^{\kappa \lambda \mu \nu} R_{\kappa \lambda \mu \nu}-4 R^{\kappa \lambda} R_{\kappa \lambda}+R^{2}\right)
$$

where $R_{\kappa \lambda \mu \nu}$ is the Riemann curvature tensor, $R_{\kappa \lambda}$ is the Ricci tensor, and $R$ is the Ricci scalar (often the topological Euler density is given without the factor $1 / 32 \pi^{2}$ (see, e.g., [52]). In fact, the terms in the parenthesis constitute the socalled "quadratic Gauss-Bonnet term" in the Lovelock gravity Lagrangian). For a general, spherically symmetric and static geometry described by the line element

$$
d s^{2}=f(r) d t^{2}-f(r)^{-1} d r^{2}-r^{2}\left(d \theta^{2}+\sin ^{2} \theta d \varphi^{2}\right)
$$

(1) becomes

$$
\mathscr{G}=\frac{4}{r^{2}}\left\{f^{\prime}(r)^{2}+[f(r)-1] f^{\prime \prime}(r)\right\}
$$

where the constant $32 \pi^{2}$ has been absorbed in $\mathscr{G}$. For constant topological density $\mathscr{G}=\alpha \neq 0$, (3) gives for the metric function

$$
f(r)=1 \pm\left(1-2 A+B r+\frac{\alpha r^{4}}{24}\right)^{1 / 2}
$$

with $A, B$ arbitrary constants. In what follows, we will keep only the negative sign of (4), as it is the one leading to black hole solutions.

The new solution derived in [50] is based on the coupling of gravity to nonlinear electromagnetic fields as described by the nonlinear generalisation of Maxwell's electrodynamics according to the Born-Infeld theory. Thus, in the chosen case of electrovacuum, the radial electric field,

$$
E(r)=\frac{r^{2}}{4 q}\left(4 R^{\mu \nu} R_{\mu \nu}-R^{2}\right)^{1 / 2}
$$

where $q$ is the electric charge, solves the Einstein-nonlinear electrodynamics coupled system with the Ricci tensor $R_{\mu \nu}$ and the Ricci scalar $R$ calculated by using the line element (2) and the metric function (4). In fact, with the values $A=1 / 2+q^{2} \Lambda / 3, B=4 M \Lambda / 3$, and $\alpha=8 \Lambda^{2} / 3$, with $\Lambda$ being the cosmological constant and $M$ being the mass of the black hole, the line element (2) with the metric function (4) becomes

$$
\begin{aligned}
d s^{2}= & {\left[1-\sqrt{\frac{4 M \Lambda r}{3}+\frac{\Lambda^{2} r^{4}}{9}-\frac{2 q^{2} \Lambda}{3}}\right] d t^{2} } \\
& -\left[1-\sqrt{\frac{4 M \Lambda r}{3}+\frac{\Lambda^{2} r^{4}}{9}-\frac{2 q^{2} \Lambda}{3}}\right]^{-1} d r^{2} \\
& -r^{2}\left(d \theta^{2}+\sin ^{2} \theta d \varphi^{2}\right)
\end{aligned}
$$

describing a Reissner-Nordström-de Sitter black hole spacetime geometry.

Now one can distinguish between two different cases, namely, the massive case $(M \neq 0)$ and the massless case $(M=0)$. In the first case, (6), when $q=0$ and $\Lambda>0$, shows that the geometry is regular everywhere except at the origin $r=0$. However, this case has no particular interest 
from the electrodynamic viewpoint. Black hole solutions with zero mass were proposed as a conjecture by A. Strominger [53] in order to explain conifold singularities in the context of string theory. In particular, the ten-dimensional IIA (resp., IIB) string theory admits black D2- (resp., D3-) brane solutions with a mass proportional to their area. After applying a Calabi-Yau compactification these solutions may wrap around minimal 2-surfaces (resp., 3-surfaces) in the Calabi-Yau space and they appear as four-dimensional black holes. As the area of the surface around which they wrap is let to go to zero, the corresponding extremal black holes become massless, topologically stable, structures. In fact, the existence of stable black hole solutions with zero ADM mass was shown in [54] although their relation to the massless solutions suggested in [53] is still not clarified. Indeed, since then there has been an increasing interest in massless black hole solutions (see, e.g., $[55,56]$ and references therein). Recently, massless black hole solutions were obtained from the dyonic black hole solution of the Einstein-Maxwell-dilaton thery [57]. Although the black hole solution examined here does not originate from string theory we will proceed to the consideration of the massless $(M=0)$ case despite its physically dubious character.

Thus for $M=0$, the line element (6) becomes

$$
\begin{aligned}
d s^{2}= & {\left[1-\sqrt{\frac{\Lambda^{2} r^{4}}{9}-\frac{2 q^{2} \Lambda}{3}}\right] d t^{2} } \\
& -\left[1-\sqrt{\frac{\Lambda^{2} r^{4}}{9}-\frac{2 q^{2} \Lambda}{3}}\right]^{-1} d r^{2} \\
& -r^{2}\left(d \theta^{2}+\sin ^{2} \theta d \varphi^{2}\right) .
\end{aligned}
$$

Here, when $q=0$ and $\Lambda \neq 0$ the de Sitter solution is obtained, while for $q \neq 0$ but $\Lambda=0$ one gets the Minkowski solution. In the case $q \neq 0$ and $\Lambda<0$, an event horizon exists and the spacetime is singular at the origin $r=0$, while the electric field is everywhere regularisable. An overall detailed study of the black hole's behavior in the massive as well as in the massless case is presented in [50].

\section{Einstein and Møller \\ Energy-Momentum Complexes}

In this section we outline the definitions of the Einstein and Møller energy-momentum complexes.

The expression for the Einstein energy-momentum complex [11] in the case of a $(3+1)$-dimensional gravitational background was later found to be given by

$$
\theta_{\nu}^{\mu}=\frac{1}{16 \pi} h_{v, \lambda}^{\mu \lambda}
$$

The Freud superpotentials $h_{v}^{\mu \lambda}$ in (8) are calculated by the compact formula (found by Landau and Lifshitz)

$$
h_{\nu}^{\mu \lambda}=\frac{1}{\sqrt{-g}} g_{\nu \sigma}\left[-g\left(g^{\mu \sigma} g^{\lambda \kappa}-g^{\lambda \sigma} g^{\mu \kappa}\right)\right]_{, \kappa}
$$

and satisfy the antisymmetric property

$$
h_{v}^{\mu \lambda}=-h_{v}^{\lambda \mu} .
$$

We notice that in the Einstein prescription the local conservation law is respected:

$$
\theta_{v, \mu}^{\mu}=0
$$

Consequently, the energy and momentum can be calculated in Einstein's prescription by

$$
P_{v}=\iiint \theta_{\nu}^{0} d x^{1} d x^{2} d x^{3} .
$$

Here, $\theta_{0}^{0}$ and $\theta_{i}^{0}$ represent the energy and momentum density components, respectively.

Applying Gauss' theorem, the energy-momentum reads

$$
P_{\mu}=\frac{1}{16 \pi} \iint h_{\mu}^{0 i} n_{i} d S
$$

with $n_{i}$ being the outward unit normal vector over the surface $d S$. In (13) the component $P_{0}$ represents the energy.

According to [16], the Møller energy-momentum complex is

$$
\mathscr{J}_{\nu}^{\mu}=\frac{1}{8 \pi} M_{\nu, \lambda}^{\mu \lambda}
$$

with the Møller superpotentials $M_{v}^{\mu \lambda}$ given by

$$
M_{\nu}^{\mu \lambda}=\sqrt{-g}\left(\frac{\partial g_{v \sigma}}{\partial x^{\kappa}}-\frac{\partial g_{\kappa \kappa}}{\partial x^{\sigma}}\right) g^{\mu \kappa} g^{\lambda \sigma} .
$$

The Møller superpotentials $M_{v}^{\mu \lambda}$ satisfy the antisymmetric property

$$
M_{\nu}^{\mu \lambda}=-M_{\nu}^{\lambda \mu}
$$

As in the case of the Einstein prescription, in the Møller prescription the local conservation law is also satisfied:

$$
\frac{\partial \mathscr{g}_{v}^{\mu}}{\partial x^{\mu}}=0
$$

In (17) the component $\mathscr{J}_{0}^{0}$ represents the energy density and $\mathscr{F}_{i}^{0}$ gives the momentum density components.

For the Møller energy-momentum complex, the energymomentum distributions are given by

$$
P_{\nu}=\iiint \mathscr{F}_{\nu}^{0} d x^{1} d x^{2} d x^{3} .
$$

In particular, the energy distribution can be computed by

$$
E=\iiint \mathscr{J}_{0}^{0} d x^{1} d x^{2} d x^{3}
$$

Using Gauss' theorem one gets

$$
P_{\nu}=\frac{1}{8 \pi} \iint M_{\nu}^{0 i} n_{i} d S,
$$

where, again, $n_{i}$ is the outward unit normal vector over the surface $d S$. 


\section{Energy and Momentum Distribution for the Black Hole Solution with Constant Topological Euler Density}

To compute the energy and momenta with the Einstein energy-momentum complex, we have to transform the metric given by the line element (6) in Schwarzschild Cartesian coordinates by using the coordinate transformation $x=$ $r \sin \theta \cos \varphi, y=r \sin \theta \sin \varphi, z=r \cos \theta$. Thus, we obtain a new form for the line element:

$$
\begin{aligned}
d s^{2}= & f(r) d t^{2}-\left(d x^{2}+d y^{2}+d z^{2}\right) \\
& -\frac{f^{-1}(r)-1}{r^{2}}(x d x+y d y+z d z)^{2} .
\end{aligned}
$$

In Schwarzschild Cartesian coordinates for $v=0,1,2,3$ and $i=1,2,3$ we find the following vanishing components of the superpotentials $h_{v}^{0 i}$ :

$$
\begin{aligned}
& h_{1}^{01}=h_{1}^{02}=h_{1}^{03}=0, \\
& h_{2}^{01}=h_{2}^{02}=h_{2}^{03}=0, \\
& h_{3}^{01}=h_{3}^{02}=h_{3}^{03}=0 .
\end{aligned}
$$

In order to compute the nonvanishing components of the superpotentials in the Einstein prescription we use (9) and we obtain the following expressions:

$$
\begin{aligned}
& h_{0}^{01}=\frac{2 x}{r^{2}} \sqrt{\frac{4 M \Lambda r}{3}+\frac{\Lambda^{2} r^{4}}{9}-\frac{2 q^{2} \Lambda}{3}}, \\
& h_{0}^{02}=\frac{2 y}{r^{2}} \sqrt{\frac{4 M \Lambda r}{3}+\frac{\Lambda^{2} r^{4}}{9}-\frac{2 q^{2} \Lambda}{3}}, \\
& h_{0}^{03}=\frac{2 z}{r^{2}} \sqrt{\frac{4 M \Lambda r}{3}+\frac{\Lambda^{2} r^{4}}{9}-\frac{2 q^{2} \Lambda}{3}} .
\end{aligned}
$$

With the aid of the line element (21), the expression for the energy given by (13), and the expressions (23)-(25) for the superpotentials, we get the energy distribution for the examined black hole in the Einstein prescription:

$$
E_{E}=\frac{r}{2} \sqrt{\frac{4 M \Lambda r}{3}+\frac{\Lambda^{2} r^{4}}{9}-\frac{2 q^{2} \Lambda}{3}} .
$$

In order to calculate the momentum components we employ (13) and (22) and performing the calculations we find that all the momenta vanish:

$$
P_{x}=P_{y}=P_{z}=0 .
$$

In the Møller prescription we perform the calculations in Schwarzschild coordinates $\{t, r, \theta, \varphi\}$ with the aid of the line element (6) and we find only one nonvanishing superpotential:

$$
M_{0}^{01}=-\frac{1}{6} \frac{12 M \Lambda+4 \Lambda^{2} r^{3}}{\sqrt{12 M \Lambda r+\Lambda^{2} r^{4}-6 q^{2} \Lambda}} r^{2} \sin \theta
$$

TABLE 1: Limiting behavior of the energy of the massive $(M \neq 0)$ black hole solution in the Einstein and Møller prescriptions.

\begin{tabular}{lcc}
\hline Energy & $r \longrightarrow \infty$ & $q=0$ \\
\hline$E_{E}$ & $\infty$ & $\frac{r}{2} \sqrt{\frac{4 M \Lambda r}{3}+\frac{\Lambda^{2} r^{4}}{9}}$ \\
\hline$E_{M}$ & $-\infty$ & $-\frac{r^{2}}{12} \frac{12 M \Lambda+4 \Lambda^{2} r^{3}}{\sqrt{12 M \Lambda r+\Lambda^{2} r^{4}}}$ \\
\hline
\end{tabular}

Using the above expression for the superpotential and the expression for the energy obtained from (20), we get the energy in the Møller prescription:

$$
E_{M}=-\frac{r^{2}}{12} \frac{12 M \Lambda+4 \Lambda^{2} r^{3}}{\sqrt{12 M \Lambda r+\Lambda^{2} r^{4}-6 q^{2} \Lambda}} .
$$

Finally, all the momenta are found to be zero:

$$
P_{r}=P_{\theta}=P_{\varphi}=0 \text {. }
$$

\section{Discussion}

Our paper focuses on the analysis of the energy-momentum localization for a new four-dimensional, spherically symmetric, static, and charged black hole spacetime geometry with constant nonzero topological Euler density, given by the line element (6). The solution describes a Reissner-Nordström-de Sitter spacetime geometry as the result of the coupling of Einstein gravity with nonlinear electrodynamics of the Born-Infeld type. For $q=0$ the solution has a near-de Sitter behavior, while for $\Lambda>0$ the solution is regular everywhere except at the origin $r=0$. To perform our study we use the Einstein and Møller energy-momentum complexes. The calculations provide the well-defined expressions (26) and (29) for the energy distribution in both prescriptions. These energy distributions depend on the mass $M$ and the charge $q$ of the black hole, the cosmological constant $\Lambda$, and the radial coordinate $r$, while in both energy-momentum complexes all the momenta vanish.

In order to study the limiting behavior of the energy distributions obtained by the Einstein and Møller prescriptions, we consider the energy for $r \longrightarrow \infty$ in the uncharged case $q=0$ for the massive $(M \neq 0)$ black hole and for $r \longrightarrow \infty$, $\Lambda=0$, and $q=0$ for the massless $(M=0)$ black hole.

Starting with the massive black hole $(M \neq 0)$ the results for the limiting cases $r \longrightarrow \infty$ and $q=0$ are presented in Table 1.

For the charged $(q \neq 0)$ black hole without cosmological constant $(\Lambda=0)$, the spacetime geometry becomes the Minkowski geometry. If the black hole is uncharged $(q=0)$ and the cosmological constant is nonzero $(\Lambda>0)$, then the spacetime is regular everywhere except at the origin $r=0$, as it is inferred from the calculation of the curvature invariants (Kretschmann and Ricci) in [50]. Indeed, the fact that, despite the regular behavior of the metric, the energy diverges at infinity in both prescriptions can possibly be attributed to the de Sitter like asymptotic behavior of the solution. The 

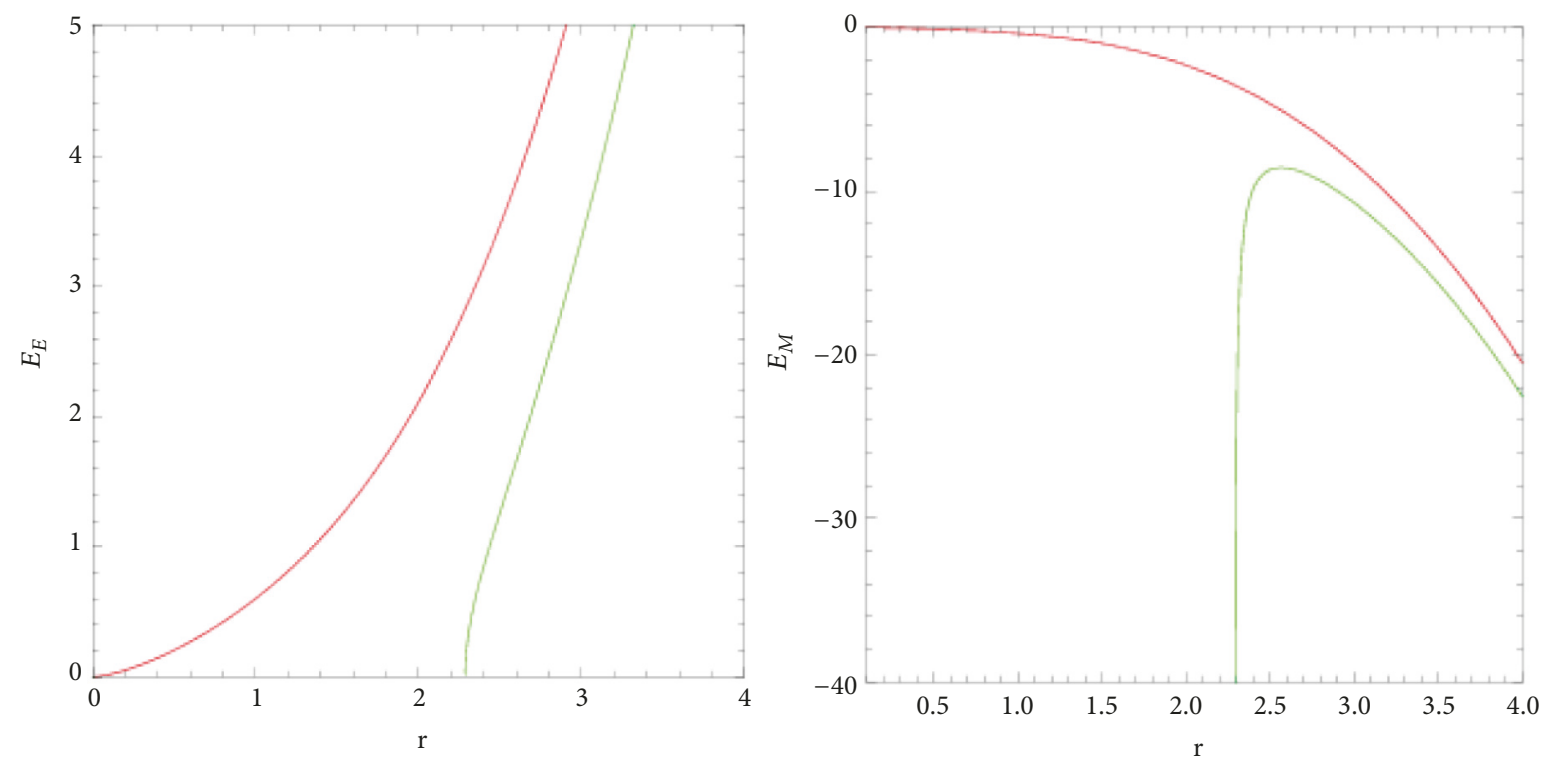

Figure 1: Evolution of the Einstein energy $E_{E}$ (left) and of the Møller energy $E_{M}$ (right) with respect to the $r$ coordinate for the massive and charged black hole. In both cases we have chosen $M=1$ and $q=1$, while the red line and the green line correspond to $\Lambda=1$ and $\Lambda=-1$, respectively.

evolution of the Einstein energy and of the Møller energy with respect to the $r$ coordinate, as given by (26) and (29), is presented for $\Lambda>0$ and $\Lambda<0$ in Figure 1. In fact, the Møller energy given by (29) is negative for a large range of values for $r$ and the parameters $M, \Lambda$, and $q$. The observed negativity of the energy might sound a note of warning regarding the physical interpretation of this result and, consequently, the merits of the Møller complex despite the physically acceptable results obtained by the latter in many other cases. Furthermore, if one considers the behavior of the energy regarding $r$, one concludes that the fourth-degree polynomial $12 M \Lambda r+\Lambda^{2} r^{4}-6 q^{2} \Lambda$ in the denominator of (29) vanishes for several sets of values for the parameters $M, \Lambda$, and $q$ with its roots consisting in two real and two complex conjugate solutions. As a result, at finite distances from the black hole the Einstein energy, given by (26), vanishes, while the Møller energy, given by (29), diverges. Equally interesting is the vanishing of the third-degree polynomial $12 M \Lambda+4 \Lambda^{2} r^{3}$ in the nominator of (29). In this case, one real and two complex conjugate solutions are obtained for every finite value of the mass parameter $M$ and $\Lambda<0$. As a result, the Møller energy vanishes at finite distances from the black hole. We have not found any plausible explanation for this rather pathological behavior of the energy in the two prescriptions, beside the fact that the black hole solution considered is quite peculiar. Some more light could be shed on this strange state of things through the comparison of the present results with the energy calculated, for example, by the Landau-Lifshitz and the Weinberg energy-momentum complexes for the metric considered, a task left for future work.

In the massless case $(M=0)$, when the black hole is charged $(q \neq 0)$ and the cosmological constant is nonzero $(\Lambda \neq 0)$, the spacetime geometry is described by the line element (7). In fact, an event horizon appears at $r_{\mathrm{eh}}=\left[9 / \Lambda^{2}+\right.$
TABLE 2: Energy of the massless $(M=0)$ black hole solution in the Einstein and Møller prescriptions.

\begin{tabular}{lcc}
\hline Energy & $q \neq 0, \Lambda \neq 0$ & $q=0, \Lambda \neq 0$ \\
\hline$E_{E}$ & $\frac{r}{2} \sqrt{\frac{\Lambda^{2} r^{4}}{9}-\frac{2 q^{2} \Lambda}{3}}$ & $\frac{r}{2} \sqrt{\frac{\Lambda^{2} r^{4}}{9}}$ \\
\hline$E_{M}$ & $-\frac{1}{3} \frac{\Lambda^{2} r^{5}}{\sqrt{\Lambda^{2} r^{4}-6 q^{2} \Lambda}}$ & $-\frac{1}{3} \frac{\Lambda^{2} r^{5}}{\sqrt{\Lambda^{2} r^{4}}}$ \\
\hline
\end{tabular}

$\left.6 q^{2} / \Lambda\right]^{1 / 4}$ for a negative cosmological constant $\Lambda<-3 /\left(2 q^{2}\right)$. Further, when $\Lambda>0$, at the position $r_{s}=\left(6 q^{2} / \Lambda\right)^{1 / 4}$ a singularity appears, while, when $\Lambda<0$, this singularity can be avoided but there appears another singularity at $r=0$ (see [50]). If this massless black hole has no charge $(q=0)$ but the cosmological constant in nonzero $(\Lambda \neq 0)$, then a de Sitter spacetime geometry is obtained:

$$
\begin{aligned}
d s^{2}= & \left(1-\sqrt{\frac{\Lambda^{2} r^{4}}{9}}\right) d t^{2}-\left(1-\sqrt{\frac{\Lambda^{2} r^{4}}{9}}\right)^{-1} d r^{2} \\
& -r^{2}\left(d \theta^{2}+\sin ^{2} \theta d \varphi^{2}\right),
\end{aligned}
$$

with a cosmological horizon appearing at $r_{\mathrm{ch}}=\sqrt{3 / \Lambda}$. The energy of the massless black hole for the charged and the uncharged case, computed in the Einstein and Møller prescriptions, is presented in Table 2 .

In Table 3 we present the limiting behavior of the Einstein energy and the Møller energy for the charged and the uncharged massless $(M=0)$ black hole as $r \longrightarrow \infty$. In both cases the cosmological constant is nonzero. 
TABLE 3: Limiting behavior of the energy of the massless black hole solution in the Einstein and Møller prescriptions.

\begin{tabular}{lcc}
\hline Energy & $q \neq 0, \Lambda \neq 0, r \longrightarrow \infty$ & $q=0, \Lambda \neq 0, r \longrightarrow \infty$ \\
\hline$E_{E}$ & $\infty$ & $\infty$ \\
$E_{M}$ & $-\infty$ & $-\infty$ \\
\hline
\end{tabular}

According to the obtained results, we come to the conclusion that the Einstein and Møller energy-momentum complexes may provide an instructive tool for the study of the energy-momentum localization of gravitating systems, although in this work we cannot reach a definite answer to the problem of localization. That being said, the investigation of the problem of the energy-momentum localization in the context of the black hole solution considered here, by applying other energy-momentum complexes as well as the teleparallel equivalent of general relativity (TEGR), is planned as a future perspective.

\section{Data Availability}

No data were used to support this study.

\section{Conflicts of Interest}

The authors declare that they have no conflicts of interest.

\section{Acknowledgments}

Farook Rahaman and Surajit Chattopadhyay are grateful to the Inter-University Centre for Astronomy and Astrophysics (IUCAA), India, for providing the Associateship Programme. Farook Rahaman is thankful to DST, Govt. of India, for providing financial support under the SERB programme. Irina Radinschi is indebted to Prof. Rodica Tudorache and Prof. Dorel Fetcu from the Department of Mathematics and Informatics of the "Gheorghe Asachi" Technical University, Iasi, Romania, for their invaluable assistance.

\section{References}

[1] L. Bel, "Définition d'une densité d'énergie et d'un état de radiation totale généralisée," Comptes Rendus de l'Académie des Sciences, vol. 246, pp. 3015-3018, 1958.

[2] I. Robinson, "On the Bel-Robinson tensor," Classical and Quantum Gravity, vol. 14, no. 1A, pp. A331-A333, 1997.

[3] M. A. G. Bonilla and J. M. M. Senovilla, "Some properties of the BEL and BEL-Robinson tensors," General Relativity and Gravitation, vol. 29, no. 1, pp. 91-116, 1997.

[4] J. M. Senovilla, "Super-energy tensors," Classical and Quantum Gravity, vol. 17, no. 14, pp. 2799-2841, 2000.

[5] J. D. Brown and J. W. York Jr., "Quasilocal energy and conserved charges derived from the gravitational action," Physical Review D: Particles, Fields, Gravitation and Cosmology, vol. 47, no. 4, pp. 1407-1419, 1993.

[6] S. A. Hayward, "Quasilocal gravitational energy," Physical Review D: Particles, Fields, Gravitation and Cosmology, vol. 49, no. 2, pp. 831-839, 1994.
[7] C.-M. Chen and J. M. Nester, "Quasilocal quantities for general relativity and other gravity theories," Classical and Quantum Gravity, vol. 16, no. 4, pp. 1279-1304, 1999.

[8] C.-C. M. Liu and S.-T. Yau, "Positivity of quasilocal mass," Physical Review Letters, vol. 90, no. 23, Article ID 231102, 2003.

[9] L. Balart, "Quasilocal energy, Komar charge and horizon for regular black holes," Physics Letters B, vol. 687, no. 4-5, pp. 280285, 2010.

[10] L. B. Szabados, "Quasi-local energy-momentum and angular momentum in general relativity," Living Reviews in Relativity, vol. 12, article 4, 2009.

[11] A. Einstein, "On the general theory of relativity," Sitzungsberichte der Königlich Preussischen Akademie der Wissenschaften, vol. 47, pp. 778-786, 1915, Addendum: Sitzungsberichte der Königlich Preussischen Akademie der Wissenschaften., vol. 47, p. 799, 1915.

[12] A. Trautman, "Conservation Laws in General Relativity," in Gravitation: An Introduction to Current Research, L. Witten, Ed., pp. 169-198, John Wiley \& Sons, New York, NY, USA, 1962.

[13] L. D. Landau and E. M. Lifshitz, The Classical Theory of Fields, Addison-Wesley, Reading, Mass, USA, 1951.

[14] A. Papapetrou, "Einstein's theory of gravitation and flat space," Proceedings of the Royal Irish Academy, vol. 52, pp. 11-23, 1948.

[15] P. G. Bergmann and R. Thomson, "Spin and angular momentum in general relativity," Physical Review A: Atomic, Molecular and Optical Physics, vol. 89, no. 2, pp. 400-407, 1953.

[16] C. Møller, "On the localization of the energy of a physical system in the general theory of relativity," Annals of Physics, vol. 4, no. 4, pp. 347-371, 1958.

[17] S. Weinberg, Gravitation and Cosmology: Principles and Applications of General Theory of Relativity, John Wiley \& Sons, New York, NY, USA, 1972.

[18] A. K. Sinha, G. K. Pandey, A. K. Bhaskar et al., "Effective gravitational mass of the Ayón-Beato and García metric," Modern Physics Letters A, vol. 30, no. 25, 1550120, 12 pages, 2015.

[19] S. K. Tripathy, B. Mishra, G. K. Pandey, A. K. Singh, T. Kumar, and S. S. Xulu, "Energy and momentum of Bianchi type VI h universes," Advances in High Energy Physics, vol. 2015, Article ID 705262, 8 pages, 2015.

[20] M. Saleh, B. B. Thomas, and T. C. Kofane, "Energy distribution and thermodynamics of the quantum-corrected Schwarzschild black hole," Chinese Physics Letters, vol. 34, no. 8, Article ID 080401, 2017.

[21] P. K. Sahoo, K. L. Mahanta, D. Goit et al., "Einstein energymomentum complex for a phantom black hole metric," Chinese Physics Letters, vol. 32, no. 2, Article ID 020402, 2015.

[22] I.-C. Yang, "Some characters of the energy distribution for a charged wormhole," Chinese Journal of Physics, vol. 53, no. 6, Article ID 110108, pp. 1-4, 2015.

[23] I. Radinschi and T. Grammenos, "Møller's energy-momentum complex for a spacetime geometry on a noncommutative curved D3-brane," International Journal of Theoretical Physics, vol. 47, no. 5, pp. 1363-1372, 2008.

[24] I.-C. Yang, C.-L. Lin, and I. Radinschi, “The energy of regular black hole in general relativity coupled to nonlinear electrodynamics," International Journal of Theoretical Physics, vol. 48, no. 1, pp. 248-255, 2009.

[25] E. C. Vagenas, "Energy distribution in 2D stringy black hole backgrounds," International Journal of Modern Physics A, vol. 18, no. 31, pp. 5781-5794, 2003. 
[26] I. Radinschi, F. Rahaman, and A. Banerjee, "On the energy of Hořava-Lifshitz black holes," International Journal of Theoretical Physics, vol. 50, no. 9, pp. 2906-2916, 2011.

[27] I. Radinschi, F. Rahaman, T. Grammenos, and S. Islam, "Einstein and Møller Energy-Momentum Complexes for a New Regular Black Hole Solution with a Nonlinear Electrodynamics Source," Advances in High Energy Physics, vol. 2016, Article ID 9049308, 2016.

[28] I. Radinschi, T. Grammenos, F. Rahaman et al., "EnergyMomentum for a Charged Nonsingular Black Hole Solution with a Nonlinear Mass Function," Advances in High Energy Physics, vol. 2017, Article ID 7656389, 10 pages, 2017.

[29] M. Abdel-Megied and R. M. Gad, "Møller's Energy in the Kantowski-Sachs Space-Time," Advances in High Energy Physics, vol. 2010, Article ID 379473, 6 pages, 2010.

[30] M. Sharif and M. Azam, "Energy-momentum distribution: some examples," International Journal of Modern Physics A, vol. 22, no. 10, pp. 1935-1951, 2007.

[31] T. Multamäki, A. Putaja, E. C. Vagenas, and I. Vilja, "Energymomentum complexes in $f(R)$ theories of gravity," Classical and Quantum Gravity, vol. 25, no. 7, Article ID 075017, 2008.

[32] L. Balart, "Energy distribution of (2+1)-dimensional black holes with nonlinear electrodynamics," Modern Physics Letters A, vol. 24, no. 34, pp. 2777-2785, 2009.

[33] A. M. Abbassi, S. Mirshekari, and A. H. Abbassi, "Energymomentum distribution in static and nonstatic cosmic string space-times," Physical Review D: Particles, Fields, Gravitation and Cosmology, vol. 78, no. 6, Article ID 064053, 9 pages, 2008.

[34] J. Matyjasek, "Some remarks on the Einstein and Møller pseudotensors for static and spherically-symmetric configurations," Modern Physics Letters A, vol. 23, no. 8, pp. 591-601, 2008.

[35] J. W. Maluf, "The teleparallel equivalent of general relativity," Annalen der Physik, vol. 525, no. 5, pp. 339-357, 2013.

[36] R. M. Gad, "On teleparallel version of stationary axisymmetric solutions and their energy contents," Astrophysics and Space Science, vol. 346, no. 2, pp. 553-557, 2013.

[37] G. G. Nashed, "Energy and momentum of a spherically symmetric dilaton frame as regularized by teleparallel gravity, Annalen der Physik, vol. 523, no. 6, pp. 450-458, 2011.

[38] G. G. L. Nashed, "Braneworld black holes in teleparallel theory equivalent to general relativity and their Killing vectors, energy, momentum and angular momentum," Chinese Physics B, vol. 19, no. 2 , p. $020401,2010$.

[39] M. Sharif and A. Jawad, "Energy contents of some well-known solutions in teleparallel gravity," Astrophysics and Space Science, vol. 331, no. 1, pp. 257-263, 2010.

[40] J. G. da Silva and S. C. Ulhoa, "On gravitational energy in conformal teleparallel gravity," Modern Physics Letters A, vol. 32, no. 21, 1750113, 10 pages, 2017.

[41] R. Penrose, "Quasilocal mass and angular momentum in general relativity," Proceedings of the Royal Society A Mathematical, Physical and Engineering Sciences, vol. 381, no. 1780, pp. 53-63, 1982.

[42] K. P. Tod, "Some examples of Penrose's quasi-local mass construction," Proceedings of the Royal Society A Mathematical, Physical and Engineering Sciences, vol. 388, no. 1795, pp. 457477, 1983

[43] J. M. Aguirregabiria, A. Chamorro, and K. S. Virbhadra, "Energy and angular momentum of charged rotating black holes," General Relativity and Gravitation, vol. 28, no. 11, pp. 1393-1400, 1996
[44] S. S. Xulu, "Bergmann-Thomson energy-momentum complex for solutions more general than the Kerr-Schild class," International Journal of Theoretical Physics, vol. 46, no. 11, pp. 29152922, 2007.

[45] K. S. Virbhadra, "Naked singularities and Seifert's conjecture," Physical Review D: Particles, Fields, Gravitation and Cosmology, vol. 60, no. 10, Article ID 104041, 6 pages, 1999.

[46] G. Sun, C.-M. Chen, J.-L. Liu, and J. M. Nester, "An optimal choice of reference for the quasi-local gravitational energy and angular momentum," Chinese Journal of Physics, vol. 52, no. 1 , part 1, pp. 111-125, 2014.

[47] C.-M. Chen and J. M. Nester, "A symplectic Hamiltonian derivation of the quasilocal energy-momentum for general relativity," Gravitation \& Cosmology, vol. 6, no. 4, pp. 257-270, 2000.

[48] C.-M. Chen, J. M. Nester, and R.-S. Tung, "Gravitational energy for GR and Poincaré gauge theories: a covariant Hamiltonian approach," International Journal of Modern Physics D: Gravitation, Astrophysics, Cosmology, vol. 24, no. 11, 1530026, 73 pages, 2015.

[49] J. M. Nester, C. M. Chen, J.-L. Liu, and G. Sun, "A reference for the covariant Hamiltonian boundary term," in Relativity and Gravitation-100 years after Einstein in Prague, J. Bicák and T. Ledvinka, Eds., pp. 177-184, Springer, 2014

[50] P. Bargueño and E. C. Vagenas, "Black holes with constant topological Euler density," European Physics Letters, vol. 115, no. 6, p. 60002, 2016.

[51] P. Gilkey, J. H. Park, and R. Vázquez-Lorenzo, Aspects of Differential Geometry I, chapter 3, Morgan \& Claypool Publishers, 2015.

[52] M. Ammon and J. Erdmenger, Gauge/Gravity Duality - Foundations and Applications, Cambridge University Press, 2015.

[53] A. Strominger, "Massless black holes and conifolds in string theory," Nuclear Physics. B. Theoretical, Phenomenological, and Experimental High Energy Physics. Quantum Field Theory and Statistical Systems, vol. 451, no. 1-2, pp. 96-108, 1995.

[54] K. Behrndt, "About a class of exact string backgrounds," Nuclear Physics. B. Theoretical, Phenomenological, and Experimental High Energy Physics. Quantum Field Theory and Statistical Systems, vol. 455, no. 1-2, pp. 188-210, 1995.

[55] R. Emparan, "Massless black hole pairs in string theory," Physics Letters. B. Particle Physics, Nuclear Physics and Cosmology, vol. 387, no. 4, pp. 721-726, 1996.

[56] U. Nucamendi and D. Sudarsky, "Black holes with zero mass," Classical and Quantum Gravity, vol. 17, no. 19, pp. 4051-4058, 2000.

[57] P. Goulart, "Massless black holes and charged wormholes in string theory," 2016, https://arxiv.org/abs/1611.03164 

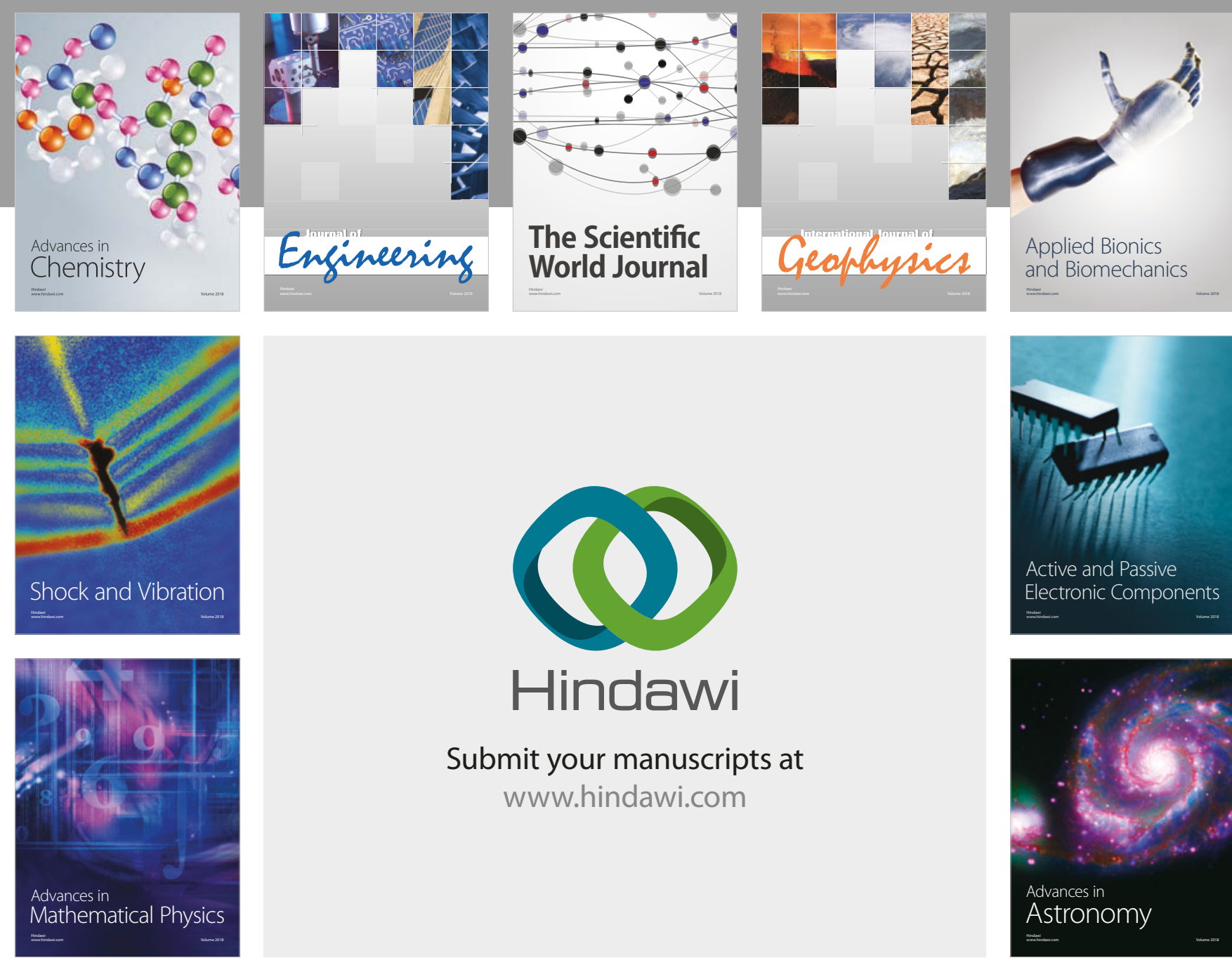

Submit your manuscripts at

www.hindawi.com

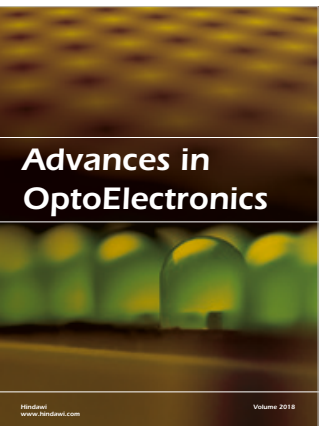

\section{Rotcting Machinery}
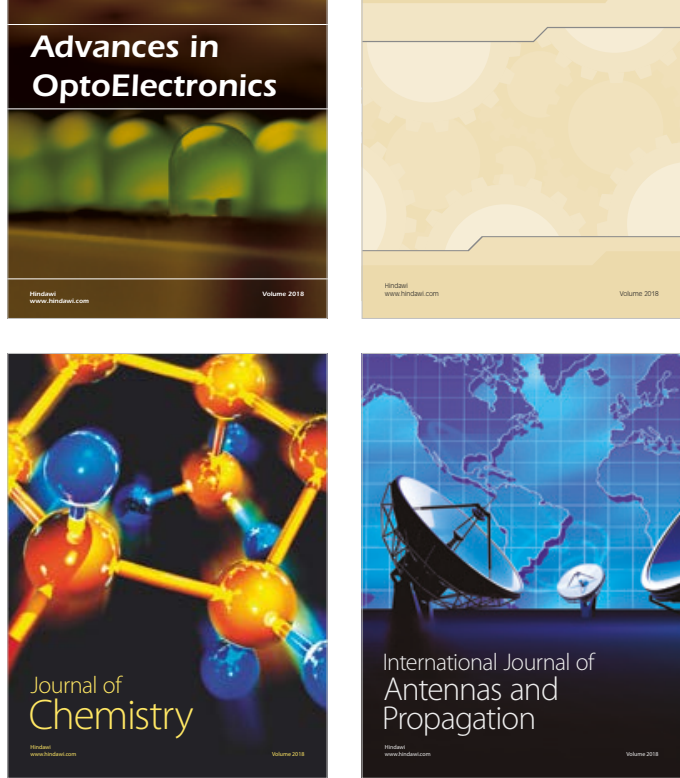

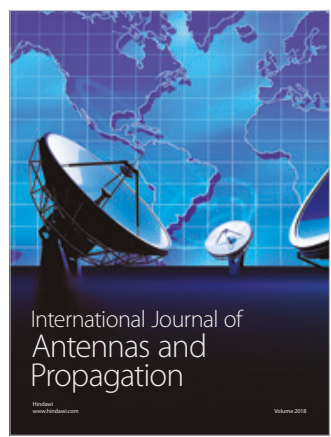

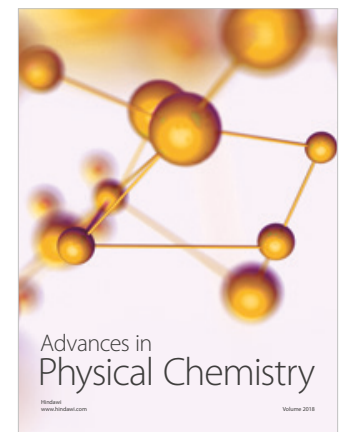

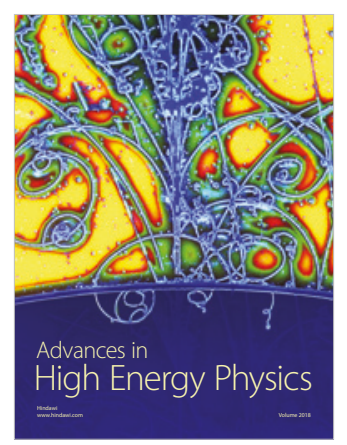

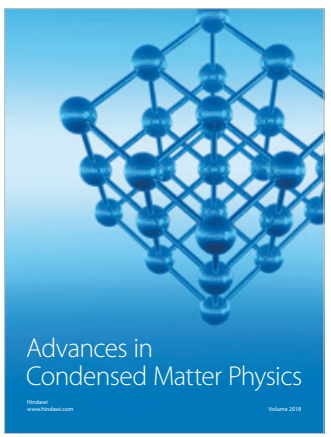

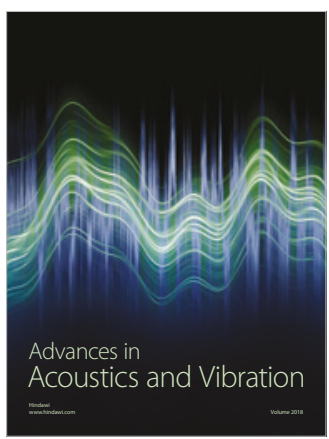

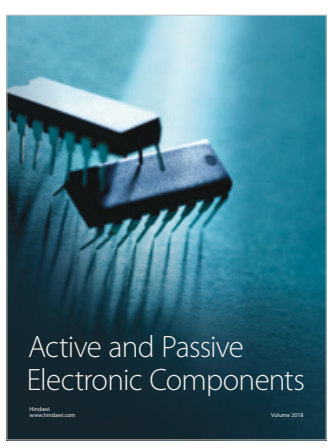
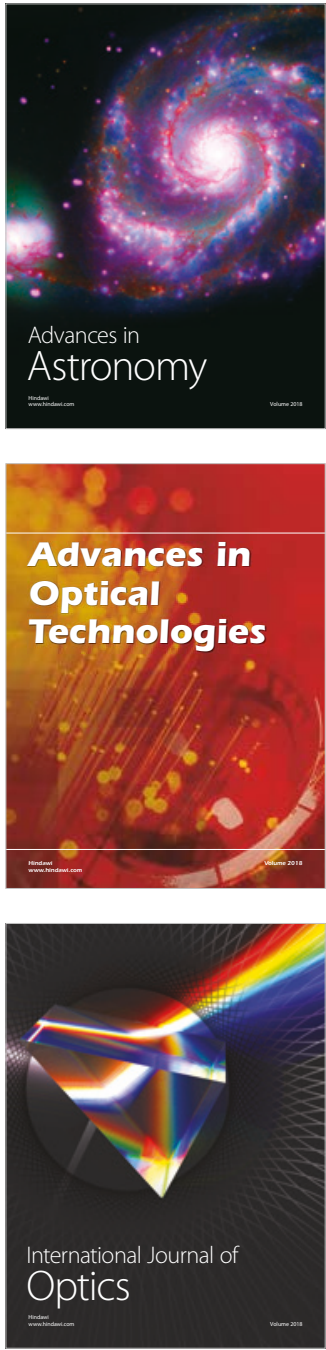